\title{
Trapping of trace gases by growing ice surfaces including surface-saturated adsorption
}

\author{
B. Kärcher, ${ }^{1}$ J. P. D. Abbatt, ${ }^{2}$ R. A. Cox, ${ }^{3}$ P. J. Popp, ${ }^{4,5}$ and C. Voigt ${ }^{1}$ \\ Received 5 February 2009; revised 11 May 2009; accepted 15 May 2009; published 15 July 2009.
}

[1] Key steps leading to the uptake of trace gases into atmospheric cloud ice particles include gas phase diffusion of trace gas molecules toward growing ice crystals and the kinetics of molecular interactions at the ice surfaces. In the case of nitric acid, laboratory studies and airborne field observations indicate uptake in growing ice films and cirrus ice particles above the thermodynamic solubility limit. This implies that uptake of nitric acid molecules on growing ice surfaces (trapping) controls the nitric acid content in ice particles residing in supersaturated air. A previous trapping model is updated to include effects of surface-saturated adsorption. Exact analytical solutions to the problem are derived to enable the calculation of the amount of vapor trapped for a given ice growth rate, assuming Langmuir-type adsorption isotherms. Those solutions are provided in terms of trapping efficiencies and equivalent uptake coefficients, net vapor fluxes toward ice crystals or ice films, steady state molar ratios of trapped molecules in the ice phase, and gas-ice partitioning factors. The trapping model includes the underlying adsorption equilibrium model asymptotically for nongrowing ice particles. Comparisons to laboratory and field measurements of nitric acid uptake are carried out. Observed dependences of uptake as a function of nitric acid partial pressure, ice growth rate, and temperature are reproduced fairly well. Nitric acid molar ratios in cirrus ice are neither controlled purely by adsorption nor purely by gas phase diffusion, underscoring the importance of using the trapping concept to interpret these observations. These results also suggest further mechanisms that enhance the nitric acid content in cirrus ice at the tropical tropopause at very low temperatures. A discussion of potential model improvements outlines the physical conditions in which the updated model describes trapping well and leads to further insight into the physical nature of the trapping process.

Citation: Kärcher, B., J. P. D. Abbatt, R. A. Cox, P. J. Popp, and C. Voigt (2009), Trapping of trace gases by growing ice surfaces including surface-saturated adsorption, J. Geophys. Res., 114, D13306, doi:10.1029/2009JD011857.

\section{Introduction}

[2] Equilibrium thermodynamics yields solubilities determining the composition of solid solutions of trace gases in ice. Those equilibria develop over long timescales as diffusion coefficients in solids are very slow [Thibert and Dominé, 1998]. The chemical analysis of ice core samples serves as a prominent example for the application of the equilibrium solubility concept.

[3] Trapping refers to the irreversible, nonreactive uptake of trace gases by solid particles which grow due to an

\footnotetext{
${ }^{1}$ Deutsches Zentrum für Luft- und Raumfahrt, Institut für Physik der Atmosphäre, Oberpfaffenhofen, Germany.

${ }^{2}$ Department of Chemistry, University of Toronto, Toronto, Ontario, Canada.

${ }^{3}$ Department of Chemistry, University of Cambridge, Cambridge, UK.

${ }^{4}$ Chemical Sciences Division, NOAA Earth System Research Laboratory, and Cooperative Institute for Research in Environmental Sciences, University of Colorado, Boulder, Colorado, USA.

${ }^{5}$ Now at Leeds School of Business, University of Colorado, Boulder, Colorado, USA.

Copyright 2009 by the American Geophysical Union. 0148-0227/09/2009JD011857\$09.00
}

external forcing. Molecular accommodation and adsorption constitute the first steps in the trapping process, followed by possible incorporation of the molecules in the advancing material volume. Surface adsorption models are adequate tools to study the trace gas interaction with nongrowing surfaces [Huthwelker et al., 2006; Pöschl et al., 2007]. Gas phase diffusion of trace gas molecules toward growing ice crystals in addition to surface kinetics is a prerequisite for a proper description of uptake of trace gases into atmospheric cloud ice particles [Kärcher and Basko, 2004, hereafter referred to as KB04]. The growth of ice particles is forced by supersaturation of the ice phase, which is commonly observed in upper tropospheric cirrus clouds. Trapping is therefore inherently a nonequilibrium process.

[4] Laboratory studies and airborne field observations indicate uptake of nitric acid $\left(\mathrm{HNO}_{3}\right)$ in growing ice films and cirrus ice particles above the thermodynamic solubility limit [Ullerstam et al., 2005; Popp et al., 2007], implying that trapping is operative and leads to more efficient uptake than adsorption and subsequent dissolution into the bulk phase. A global model study investigated the potential impact of the uptake of $\mathrm{HNO}_{3}$ on ice on the nitrogen oxide 

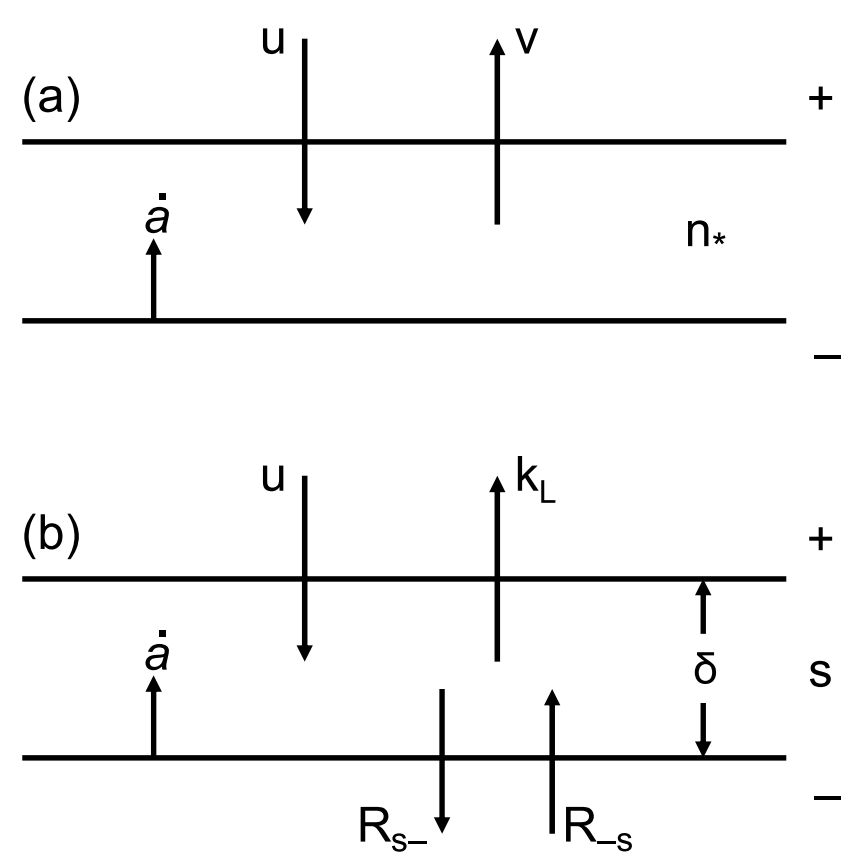

Figure 1. Schematics describing gas uptake by an ice surface growing at rate $\dot{a}=d a / d t$. Molecules diffusing through air and arriving at the particle with a mean thermal speed $u$ become adsorbed. (a) The current trapping model, where adsorbed molecules leave the particle with an escape speed $v$. The surface layer remains unresolved, i.e., it is merely treated as a boundary between the near-surface gas phase $(+)$ and the near-surface bulk phase $(-)$ regions. The maximum trapped trace gas number concentration is $n *$. (b) A possible extension, where adsorbed molecules may either leave the particle according to a desorption rate $k_{L}$ or interact with the bulk ice region. Here the surface layer (label $s$ ) is resolved and associated with a thickness $\delta$, as well as forward and backward rates $R$ between the layer and the bulk. Open parameters are $v$ and $n *$ in Figure 1a and $\delta$, $R_{s-}$, and $R_{-s}$ in Figure $1 \mathrm{~b}$.

budget and on ozone [von Kuhlmann and Lawrence, 2006]. This study assumed equilibrium uptake and concluded that the largest uncertainty in quantifying this impact is likely to be linked to the actual theory describing the uptake process, rather than to uncertainties in the description of cloud ice processes.

[5] As a first step into research on the importance of trapping in cirrus conditions, our previous version of the trapping model assumed unsaturated surface adsorption [KB04]. A comprehensive comparison of results from this model with a number of airborne measurements has been very encouraging [Kärcher and Voigt, 2006, hereinafter KV06]. Many atmospheric trace species including $\mathrm{HNO}_{3}$ exhibit surface-saturated (Langmuir-type) adsorption for sufficiently large partial pressures, which tends to limit uptake; differences between dissociative and nondissociative adsorption are rarely observed experimentally for atmospheric surfaces [Ammann et al., 2008]. Together with trapping experiments carried out in the laboratory that became available recently [Ullerstam and Abbatt, 2005], this motivated us to extend our previous trapping theory.
[6] Section 2 outlines the key features of our updated trapping model. Section 3 is devoted to the formulation of the general equations and their solution, including a brief discussion of the main solution characteristics. Section 4 addresses the comparison of theoretical predictions with laboratory and field measurements of $\mathrm{HNO}_{3}$ uptake in growing ice films and cirrus ice particles. Section 5 discusses the new results in the context of atmospheric measurements and outlines potential theoretical improvements. Section 6 concludes this work.

\section{Prerequisites}

[7] Given the air temperature $T$ and pressure $P$, the diffusional growth law $\dot{a}=d a / d t$ for an ice particle with radius $a$ can be written as [Pruppacher and Klett, 1997]

$$
\dot{a}=\nu_{w} \frac{D_{w} \beta_{w}}{a} \frac{e_{i} s_{i}}{k_{B} T}, \quad \beta_{w}=\frac{a}{a+l_{w} / \alpha_{w}}
$$

with the volume $\nu_{w}$ of water $\left(\mathrm{H}_{2} \mathrm{O}\right)$ molecules in ice, the diffusion coefficient $D_{w}(P, T)$ of $\mathrm{H}_{2} \mathrm{O}$ molecules in air, Boltzmann's constant $k_{B}$, the ice supersaturation over the particle surface $s_{i}$, and the saturation vapor pressure over ice $e_{i}(T)$. The function $\beta_{w}(a)$ interpolates between the free molecular flow $\left(a \ll l_{w} / \alpha_{w}\right)$ and the diffusion $\left(a \gg l_{w} / \alpha_{w}\right)$ regime, where $l_{w}=4 D_{w} / u_{w}$ is the diffusion length scale of $\mathrm{H}_{2} \mathrm{O}$ molecules in air, $\alpha_{w}$ is the deposition coefficient of $\mathrm{H}_{2} \mathrm{O}$ molecules on ice and $u_{w}(T)$ is their mean thermal speed. In the upper troposphere, latent heat effects are small owing to the low temperatures $(T<235 \mathrm{~K})$. Further, the majority of the ice crystals are usually too small (several tens of $\mu \mathrm{m}$ ) to be substantially heated by absorption of longwave radiation or for ventilation to alter the growth rate [Gierens, 1994]. These effects are therefore neglected in equation (1). The assumption of sphericity enables a simple estimate of typical growth rates and their general dependence on particle size. It is not crucial for the discussion of the trapping mechanism.

[8] The number $N_{w}$ of $\mathrm{H}_{2} \mathrm{O}$ molecules taken up by the ice crystal surface per unit time is given by

$$
\dot{N}_{w}=4 \pi a^{2} \dot{a} / \nu_{w}
$$

with $\dot{a}$ from equation (1). Following KB04, uptake of trace gas molecules in growing ice crystals is described by a similar expression introducing the trapping efficiency $\epsilon$ :

$$
\dot{N}=4 \pi a D \epsilon n_{\infty} \geq 0, \quad \epsilon=\frac{a}{a+l / \gamma}
$$

where $n_{\infty}$ is the trace gas concentration far away from the ice particle, $\gamma$ is the uptake coefficient describing the trapping process, and $l=4 D / u ; \epsilon$ and $\gamma$ are discussed in section 3. Trapping is irreversible as long as $s_{i}>0$. If the ice particles evaporate $\left(s_{i}<0\right)$, the trace gas will leave the ice particles along with $\mathrm{H}_{2} \mathrm{O}$ [KB04, their section 2.6]. In practice, equations (2) and (3) will be complemented by additional relationships for $n_{\infty}$ and $s_{i}$ to ensure local conservation of the total numbers of trace gas and $\mathrm{H}_{2} \mathrm{O}$ molecules. 
[9] The schematic in Figure 1a displays the kinetics describing the trapping process in the current model framework. Processes occurring in the unresolved surface layer are not explicitly represented, but are assumed to lead to an effective escape velocity, $v$, of adsorbed molecules. As shown next, this simplification allows for a straightforward derivation of the trapping efficiency with only two open parameters that implicitly contain information about surface layer processes. The strategy in this work is to constrain these parameters by measurements. We comment on the necessity to consider an explicit description of surface processes with the help of Figure $1 \mathrm{~b}$ in section 5.2.

[10] In the previous model version, KB04 have used an unsaturated (linear) surface adsorption isotherm, $n_{+} \alpha u / 4=$ $n_{-} v$, where $n_{+}$and $n_{-}$denote the trace gas number densities approaching the idealized ice surface from the gas and bulk side, respectively. We replace this relationship by

$$
(1-\theta) n_{+} \alpha u / 4=\theta n_{*} v,
$$

with the maximum number density of trapped molecules, $n_{*}$, and the fractional coverage of trapped molecules, $\theta=n_{-}$/ $n_{*} \leq 1$. The term $(1-\theta)$ accounts for surface-saturated (nonlinear) adsorption, i.e., no further uptake is possible when $\theta=1$. Equation (4a) assumes that the trace gas adsorption and escape processes evolve in a quasi steady state on the timescales over which ambient temperature, trace gas partial pressure, and $\mathrm{H}_{2} \mathrm{O}$ deposition rate change. The condition in which this assumption holds is discussed in section 3.4.

[11] Rearranging equation (4a) using the ideal gas law yields the Langmuir isotherm

$$
\theta=K p_{+} /\left(1+K p_{+}\right)
$$

with the generalized equilibrium constant $K(T)$ that is inversely proportional to an escape rate $k$ :

$$
K=\frac{1}{k_{B} T} \frac{\alpha u / 4}{n_{*} v}, \quad k / \sigma=n_{*} v,
$$

where $\sigma$ denotes the surface area per adsorption site. Equation (5a) describes the equilibrium between adsorption from the gas phase and escape from the unresolved ice surface layer. If the surface layer were resolved, $k$ would represent the true desorption rate $k_{L}$, commonly used to describe reversible adsorption equilibria, that is connected to the Langmuir constant via

$$
K_{L}=\frac{1}{k_{B} T} \frac{\alpha u / 4}{k_{L} / \sigma} .
$$

[12] The escape velocity, $v$, and the maximum trapped concentration, $n_{*}$, are the two open parameters of the trapping model. They may be linked to a characteristic depth $\delta$ of the surface layer via $v=k \delta$ and $n_{*}=1 /(\delta \sigma)$, and $\delta$ can be inferred once $n_{*}$ is known. Using this definition, the fractional coverage takes its conventional form $\theta=n_{-} \delta \cdot \sigma$ as a scaled surface concentration. However, neither $\delta$ nor $k$ need to be specified in this model. Equations (4a) and (4b) would change if the adsorption process was controlled by physical mechanisms other than those underlying the Langmuir theory, e.g., by uptake into a liquid layer of supercooled solution or water [KB04, their section 5.4].

\section{Trapping Model}

\subsection{General Equations and Solution}

[13] We formulate advective and diffusive fluxes across the moving phase boundary (Figure 1a). The diffusion of a molecule toward the ice particle surface can be well approximated by a steady state profile which develops on the timescale of milliseconds [Pruppacher and Klett, 1997]. The steady state flux of molecules diffusing in air toward a spherical particle is given by $\left(n_{+}-n_{\infty}\right) D / a$. In a reference frame moving with the velocity $\dot{a}>0$ in the direction of increasing particle radius, the advective gas phase flux triggered by the moving ice surface is given by $-n_{+} \dot{a}$. Addition of the two components yields the total flux $f_{+}$:

$$
f_{+}=-n_{+} \dot{a}+\left(n_{+}-n_{\infty}\right) D / a .
$$

The corresponding flux $f_{-}$is purely advective, as we neglect diffusion into the bulk ice which is very slow and therefore not relevant given the ice growth timescales (minutes to tens of minutes) of our problem [KB04]:

$$
f_{-}=-n_{-} \dot{a} .
$$

The flux describing net adsorption of molecules at the phase boundary reads

$$
f_{*}=-n_{+} \dot{a}+\left[n_{-} v-\left(1-n_{-} / n_{*}\right) n_{+} \alpha u / 4\right],
$$

accounting for surface-saturated adsorption according to the Langmuir isotherm introduced in section 2 and replacing equation (6c) in KB04. The trapping rate follows from

$$
\dot{N}=4 \pi a^{2} n_{-} \dot{a} .
$$

[14] The trapping equations are obtained by imposing continuity of the fluxes (6a)-(6c) at the phase boundary, $f_{-}=f_{+}=f_{*}$, constituting a set of two algebraic equations for the two unknowns $\eta_{+}=n_{+} / n_{\infty}$ and $\eta_{-}=n_{-} / n_{\infty}$ :

$$
\begin{gathered}
\eta_{-}=\kappa+(1-\kappa) \eta_{+}, \\
\eta_{+}=\frac{1-\beta\left(1-\lambda \eta_{-}\right)}{1-\beta \vartheta \eta_{-}},
\end{gathered}
$$

where $\beta, \kappa, \lambda$, and $\vartheta$ are dimensionless parameters defined in Table 1 . The two parameters $\lambda=4 v /(\alpha u)$ and $\vartheta=n_{\infty} / n_{*}$ measure $v$ and $n_{*}$, respectively. We recall from section 2 that $v$ and $n_{*}$, hence $\lambda$ and $\vartheta$, do not follow directly from the trapping model, but must be constrained by suitable data. The remaining parameters $\beta$ and $\kappa$ are determined by the size and growth rate of the ice particles. 
Table 1. Dimensionless Parameters Used in the Trapping Model ${ }^{\mathrm{a}}$

\begin{tabular}{|c|c|}
\hline Parameter & Meaning \\
\hline$\beta=[1+4 D /(\alpha u a)]^{-1}$ & contains ratio of diffusion length \\
\hline$\kappa=D /(a \dot{a})$ & $\begin{array}{l}\text { ratio of gas phase diffusion speed }(D / a) \\
\text { and ice particle growth rate }(\dot{a})\end{array}$ \\
\hline$\kappa^{\prime}=\alpha u /(4 \dot{x})$ & $\begin{array}{l}\text { ratio of scaled thermal speed }(\alpha u / 4) \\
\text { and ice film growth rate }(\dot{x})\end{array}$ \\
\hline$\lambda=4 v /(\alpha u)$ & $\begin{array}{l}\text { ratio of escape speed }(v) \text { and scaled } \\
\text { thermal speed }(\alpha u / 4)\end{array}$ \\
\hline$\vartheta=n_{\infty} / n_{*}$ & $\begin{array}{l}\text { ratio of ambient and maximum trapped } \\
\text { number density }\end{array}$ \\
\hline$\zeta=\vartheta / \lambda$ & $\begin{array}{l}\text { ratio of adsorption flux }\left(n_{\infty} \alpha u / 4\right) \text { and } \\
\text { maximum escape flux }(n * v)\end{array}$ \\
\hline$\xi=v / \dot{a}=\beta \kappa \lambda /(1-\beta)$ & $\begin{array}{l}\text { ratio of escape speed and ice particle } \\
\text { growth rate }\end{array}$ \\
\hline$\xi^{\prime}=v / \dot{x}=\kappa^{\prime} \lambda$ & $\begin{array}{l}\text { ratio of escape speed and ice film } \\
\text { growth rate }\end{array}$ \\
\hline
\end{tabular}

${ }^{\mathrm{a}}$ Only $\lambda$ and $\vartheta$ contain a priori unconstrained parameters, namely $v$ and $n *$, respectively; other parameters are defined for notational convenience.

[15] Inserting equation (8b) into equation (8a) and solving for $\eta_{-}$yields the quadratic equation

$$
\eta_{-}^{2}-\frac{1+\beta \kappa \vartheta+(\kappa-1) \beta \lambda}{\beta \vartheta} \eta_{-}+\frac{1+(\kappa-1) \beta}{\beta \vartheta}=0
$$

from which we calculate the physical solution

$$
\begin{gathered}
\eta_{-}=\frac{1+\beta \kappa(\lambda+\vartheta)}{2 \beta \vartheta}\left\{1-\sqrt{1-\frac{4 \beta \vartheta(1+\beta \kappa)}{[1+\beta \kappa(\lambda+\vartheta)]^{2}}}\right\} \\
\eta_{+}=1-\eta_{-} / \kappa .
\end{gathered}
$$

Equations (9a) and (9b) assume $\kappa \gg 1$, following from the fact that a steady state gas phase diffusion profile, represented by the second term on the right side of equation (6a), establishes very quickly [KB04, their section 2.4].

[16] We inspect important limiting cases of our general solution. For most atmospheric applications, it is safe to assume $\beta \kappa \gg 1$ in equation (9a), as $\beta$ is usually of the order $0.1-1$. The opposite relation $\beta \kappa \ll 1$ can only be achieved for $\beta \ll 1$, equivalent to free molecular flow conditions.

[17] If we only impose $\vartheta \rightarrow 0$, we obtain

$$
\eta_{-} \rightarrow \frac{\beta \kappa}{1+\beta \kappa \lambda},
$$

which is our previous solution [KB04, their equation (9)]; this is expected since the Langmuir isotherm approaches the linear isotherm used in KB04 at low ambient trace gas partial pressures. For $\lambda \rightarrow 0$, we obtain

$$
\eta_{-} \rightarrow \frac{\beta \kappa}{1+\beta \kappa \vartheta}
$$

These are the general solutions in the limits of low and high partial pressures, respectively.
[18] We delineate two important regimes where trapping is either solely controlled by gas diffusion (burial) or by surface processes (adsorption and escape). The burial limit is obtained for $\beta \kappa(\lambda+\vartheta) \ll 1$, in which case

$$
\eta_{-} \rightarrow \beta \kappa .
$$

The adsorption limit requires that $\beta \kappa(\lambda+\vartheta) \gg 1$, leading to

$$
\eta_{-} \rightarrow 1 /(\lambda+\vartheta)
$$

this regime is subdivided to describe unsaturated adsorption $(\vartheta \ll \lambda), \eta_{-} \rightarrow 1 / \lambda$, and saturated adsorption $(\vartheta \gg \lambda), \eta_{-} \rightarrow$ $1 / \vartheta$, and is further examined in section 3.4.

[19] We finally introduce various convenient measures of uptake. Equations (3) and (7) define the trapping efficiency

$$
\epsilon=\eta_{-} / \kappa
$$

a comparison with equation (9b) shows that $\epsilon$ accounts for the reduction of the ambient trace gas number density $n_{\infty}$ at the ice particle surface, $n_{+}=n_{\infty}(1-\epsilon)$. The associated uptake coefficient is given by

$$
\gamma=\frac{n_{-} \dot{a}}{n_{+} u / 4}=\alpha \frac{\epsilon}{1-\epsilon} \frac{1-\beta}{\beta} .
$$

Equation (12) is identical to equation (13) in KB04 and is consistent with the second expression in our equation (3). This steady state uptake coefficient does not describe the kinetics of populating a fresh surface and achieves its upper limit value $(\gamma=\alpha)$ only for maximum trapping efficiency in the burial limit $(\epsilon=\beta)$. Instantaneous molar ratios are given by $\tilde{\mu}=N / N_{w}$ after a time-dependent solution to equations (2) and (3) is available. For many applications, it may suffice to work with steady state molar ratios, $\mu=\dot{N} / \dot{N}_{w}$ that follow from $d \tilde{\mu} / d t=0$. With equations (2) and (7), $\mu$ is given by

$$
\mu=\nu_{w} n_{\infty} \eta_{-} .
$$

The fraction of molecules remaining in the gas phase, $\phi_{\infty}=$ $N_{\infty} /\left(N_{\infty}+N\right)$, is useful for the interpretation of field observations. In our notation

$$
\phi_{\infty}=\frac{1}{1+\nu_{w} n_{w} \eta_{-}} ;
$$

$n_{w}$ is the number density of water molecules in ice particles that can be calculated from $n_{w}=\mathrm{IWC} / m_{w}$, where IWC is the cirrus ice water content (mass of ice per unit volume of air) and $m_{w}$ is the mass of an $\mathrm{H}_{2} \mathrm{O}$ molecule. In the burial limit, $\phi_{\infty}$ achieves an upper limit value

$$
\phi_{\infty} \rightarrow\left(1+\frac{D \beta}{D_{w} \beta_{w}} z\right)^{-1}
$$

that is mainly determined by the ratio $z$ of the ice water content to the moisture available for condensation (potential ice water content),

$$
z=\frac{\mathrm{IWC} / m_{w}}{e_{i} s_{i} /\left(k_{B} T\right)} .
$$


Table 2. Limiting Solutions of the Trapping Model

\begin{tabular}{|c|c|c|c|c|c|c|}
\hline & \multicolumn{3}{|c|}{ General Solution } & \multicolumn{3}{|c|}{ Planar Flow } \\
\hline & \multirow[b]{2}{*}{ Burial Limit } & \multicolumn{2}{|c|}{ Adsorption Limit } & \multirow[b]{2}{*}{ Burial Limit } & \multicolumn{2}{|c|}{ Adsorption Limit } \\
\hline & & Unsaturated & Saturated & & Unsaturated & Saturated \\
\hline$\epsilon$ & $\beta$ & $\frac{\alpha u a}{4 D} \frac{\dot{a}}{v}$ & $\frac{a \dot{a}}{D} \frac{n_{*}}{n_{\infty}}$ & 1 & $\frac{\dot{x}}{v}$ & $\frac{4 \dot{x}}{\alpha u} \frac{n_{*}}{n_{\infty}}$ \\
\hline$\gamma$ & $\alpha$ & $\alpha \frac{\dot{a}}{v}$ & $\frac{4 \dot{a}}{u} \frac{n_{*}}{n_{\infty}}$ & $\alpha$ & $\alpha \frac{\dot{x}}{v}$ & $\frac{4 \dot{x}}{u} \frac{n_{*}}{n_{\infty}}$ \\
\hline$\dot{N}$ & $4 \pi a D \beta n_{\infty}$ & $4 \pi a^{2} \dot{a} n_{\infty} \frac{\alpha u}{4 v}$ & $4 \pi a^{2} \dot{a} n_{*}$ & $n_{\infty} \frac{\alpha u}{4}$ & $n_{\infty} \frac{\alpha u \dot{x}}{4} \frac{\dot{x}}{v}$ & $\dot{x} n *$ \\
\hline$\mu$ & $\nu_{w} n_{\infty} \frac{D \beta}{a \dot{a}}=\frac{D \beta}{D_{w} \beta_{w}} \frac{p_{\infty}}{e_{i} s_{i}}$ & $\nu_{w} n_{\infty} \frac{\alpha u}{4 v}$ & $\nu_{w} n_{*}$ & $\nu_{w} n_{\infty} \frac{\alpha u}{4 \dot{x}}=\frac{\alpha u}{\alpha_{w} u_{w}} \frac{p_{\infty}}{e_{i} s_{i}}$ & $\nu_{w} n_{\infty} \frac{\alpha u}{4 v}$ & $\nu_{w} n_{*}$ \\
\hline
\end{tabular}

\subsection{Planar (Free Molecular) Flow}

[20] In a one-dimensional (planar) geometry, we assume that an ice film grows with a velocity $\dot{x}$. The number $N$ of molecules that become trapped is now given in units of molecules per unit ice surface area. The equations for this case are obtained by formally imposing $\beta \ll 1$, the free molecular flow limit, in equation (9a). Equation (9b) is superfluous, as $n_{+} \equiv n_{\infty}$ in this limit. (A partial pressure gradient does not exist.) This leads to

$$
\eta_{-}=\frac{\kappa^{\prime}}{1+\kappa^{\prime}(\lambda+\vartheta)},
$$

with $\beta \kappa=\kappa^{\prime}$ (Table 1). Similar to $\kappa, \kappa^{\prime} \gg 1$, which we used to derive equation (16) replacing equation (B2) in KB04. The trapping efficiency is given by

$$
\epsilon=\eta_{-} / \kappa^{\prime}
$$

so we have $\dot{N}=n \_\dot{x}=\left(n_{\infty} \alpha u / 4\right) \epsilon$, which is the equivalent to equation (3). The uptake coefficient is given by

$$
\gamma=\frac{n_{-} \dot{x}}{n_{\infty} u / 4}=\alpha \epsilon .
$$

[21] Equation (18) replaces equation (B3) in KB04. As before, the molar ratio is given by equation (13).

[22] The burial limit in the free molecular regime is obtained for $\kappa^{\prime}(\lambda+\vartheta) \ll 1$, yielding $\eta_{-}=\kappa^{\prime}$, i.e., $\epsilon=1$ and $\gamma=\alpha$. The corresponding adsorption limit is realized for $\kappa^{\prime}(\lambda+\vartheta) \gg 1$, hence $\eta_{-}=1 /(\lambda+\vartheta)$, identical to the general case equation (10d). We add that the free molecular growth law takes the form $\dot{x}=\nu_{w}\left(\alpha_{w} u_{w} / 4\right) e_{i} s_{i} /\left(k_{B} T\right)$, obtained from equation (1) in the limit $\beta_{w} \ll 1$.

\subsection{Main Features of Trapping}

[23] The solutions of the trapping model in the burial and adsorption limits are summarized in Table 2. In the burial limit, the trapping efficiency is largest and both $\epsilon$ and $\gamma$ take their maximum values, indicating that the absolute amount of trace gas taken up in this way $(\propto \dot{N})$, maximizes. The molar ratios scale with the relative rates of mass transfer of the trace gas and water to the ice surface, i.e., in proportion to $D \beta /\left(D_{w} \beta_{w}\right)$ and $\alpha u /\left(\alpha_{w} u_{w}\right)$ in the general case and for planar flow, respectively. Burial causes $\mu$ to decrease inversely proportional to the growth rate, because the amount of trapped vapor is limited by its ambient partial pressure while the amount of $\mathrm{H}_{2} \mathrm{O}$ taken up increases with the growth rate.
[24] Trapping is generally less efficient in the adsorption limit, where $\epsilon, \gamma$, and $\dot{N}$ scale in proportion to the ice growth rate, as does water uptake. Therefore the steady state molar ratios do not depend on the growth rate. The growing ice surface traps the amount of vapor determined by the underlying adsorption equilibrium model (section 3.4). For unsaturated surface adsorption, uptake is growth rate limited and decreases as the escape speed increases. The trapped amount scales linearly with the ambient trace gas partial pressure, as for pure burial. For surface-saturated adsorption, the trapped amount is limited by $n *$, independent of the ambient partial pressure.

[25] We show the full solutions in terms of the uptake coefficient $\gamma$ in Figure 2 for the general case (Figure 2a) and in planar flow conditions (Figure 2b). A reduction of $p_{\infty}$ caused by depletion of the trace gas phase reservoir is not taken into account. For convenience of discussion, the underlying dimensionless variables were fixed to typical values: $\alpha=0.3$ and $\beta=0.9$; and $\kappa=2.25 \times 10^{8}$ and $\kappa^{\prime}=$ $1.35 \times 10^{9}$ resulting from $D=0.75 \mathrm{~cm}^{2} / \mathrm{s}, u=3 \times 10^{4} \mathrm{~cm} / \mathrm{s}$, $a=20 \mu \mathrm{m}$, and $\dot{a}=\dot{x}=1 \mu \mathrm{m} / \mathrm{min}$. (Section 4 discusses applications of the trapping model where these parameters vary consistently with temperature.) Plotting $\gamma$ versus $\xi\left(\xi^{\prime}\right)$ varies $\dot{a}(\dot{x})$ for a given $v$ or vice versa, and different values of $\zeta$ vary $n_{\infty}$ or $p_{\infty}$ for a given $n_{*}$ (recall Table 1 ).

[26] Both solutions behave similarly. For small values of $\xi\left(\xi^{\prime}\right)$, the burial limit $\gamma \rightarrow \alpha$ is realized. For large $\xi-\left(\xi^{\prime}-\right)$ values, $\gamma$ decreases as $\xi\left(\xi^{\prime}\right)$ increases, consistent with the solutions in the adsorption limit provided in Table 2. For $\zeta \leq 0.01$, all curves collapse and represent the unsaturated adsorption regime. The transition between burial and adsorption regime occurs near $\xi=1\left(\xi^{\prime}=1\right)$, shifting to smaller values as $\zeta$ increases. For $\zeta \gg 1$ at fixed $\xi\left(\xi^{\prime}\right), \gamma$ becomes strongly reduced as $\zeta$ increases, indicating that the ice particle surface becomes saturated, shutting off any further uptake.

\subsection{Adsorption Limit}

[27] Adsorption-limited trapping is obtained for small (or zero) ice growth rates, $\dot{a} \rightarrow 0(\dot{x} \rightarrow 0)$, where $\eta_{-} \rightarrow 1 /(\lambda+$ $\vartheta)$, both in the general case and for planar flow. The ratio of escape speed to ice growth rate becomes large. Using $\theta=$ $n_{-} / n_{*}$ and the definitions from Table 1 , we obtain

$$
\theta=\eta_{-} \vartheta=\frac{\zeta}{1+\zeta}=\frac{K p_{\infty}}{1+K p_{\infty}} .
$$

This expression is consistent with the local coverage from equation (4b) that is determined by the partial pressure $p_{+}$adjacent to the ice surface, and not by $p_{\infty}$. This is 

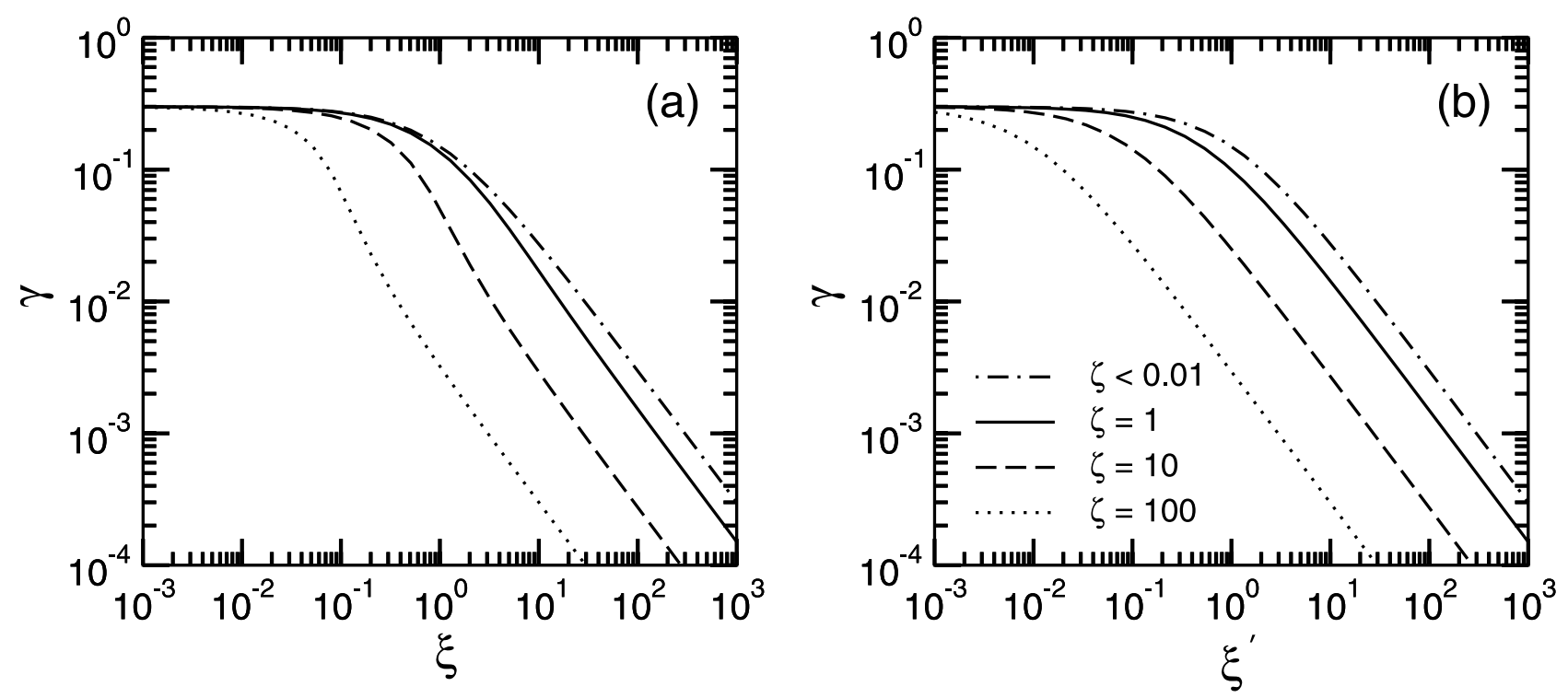

Figure 2. Uptake coefficient for trapping $\gamma$ versus (a) the ratio of escape speed to ice growth rate $\xi$ for the general solution and (b) $\xi^{\prime}$ for planar (free molecular) flow for different values of $\zeta$ interpolating between surface-saturated $(\zeta \gg 1)$ and unsaturated $(\zeta \ll 1)$ adsorption. The burial limit $\gamma \rightarrow \alpha$ is realized for $\xi \ll 1$ or $\xi^{\prime} \ll 1$, depending on $\zeta$.

easily shown by inspecting the trapping efficiency $\epsilon$ from equation (11) in the adsorption limit. We find that $\epsilon \propto \dot{a} \rightarrow 0$ or $\epsilon \propto \dot{x} \rightarrow 0$ is very small, so we have $n_{+}=n_{\infty}(1-\epsilon) \approx n_{\infty}$.

[28] In the adsorption limit, both the trace gas and the water vapor flux scale with the ice growth rate (Table 2); therefore $\mu$ does not depend on $\dot{a}$ or $\dot{x}$ and can be cast into the form

$$
\mu_{\mathrm{ads}}=\nu_{w} n_{*} \theta
$$

the asymptotic values of which are given in Table 2 for surface-saturated and unsaturated adsorption (i.e., for $\theta \rightarrow 1$ and $\theta \rightarrow \zeta \ll 1$, respectively). For small growth rates, the growing ice surface cannot trap more vapor than determined by $\theta$. Therefore the values $\mu_{\mathrm{ads}}$ are the highest possible molar ratios realized during trapping in the adsorption limit. Increasing the growth rate leads to a dilution of the particle with additional $\mathrm{H}_{2} \mathrm{O}$, reducing $\mu$ below $\mu_{\text {ads }}$. Of course, $\mu<$ $\mu_{\text {ads }}$ does not imply a lower absolute amount of trapped vapor (section 3.3).

[29] At zero ice growth rate, the adsorbed molar ratio $\mu_{\text {ads }}$ measures the number of molecules trapped in the ice surface layer, as diffusion into the bulk of the ice does not take place during the timescales of interest. By contrast, $\mu$ represents a bulk quantity, as trace gas molecules are trapped in the ice during its growth. The spatial distribution of the trace gas molecules in the ice depends on the temporal evolution of the supersaturation and the trace gas partial pressure, as well as on how the trace gas impurities are incorporated in the ice crystal lattice during growth. Nevertheless, equations (19a) and (19b) highlight that the trapping model and adsorption equilibrium isotherms for (almost) static ice surfaces do not represent rivaling concepts. Trapping includes the surface adsorption model asymptotically and extends the latter to the nonequilibrium case of growing ice surfaces, as commonly found in the atmosphere.

[30] To study whether an adsorption equilibrium establishes at the growing ice surface, as assumed in trapping model, we examine the timescale, $\tau$, over which $\theta$ relaxes to the steady state Langmuir isotherm. The rate equation governing the time evolution of $\theta$ is given by

$$
\frac{1}{4 \pi a^{2}} \frac{d \theta}{d t}=-\theta n_{*} v+(1-\theta) \frac{n_{+} \alpha u}{4}
$$

yielding equation (4a) when $d \theta / d t=0$. A conservative estimate of the steady state condition is given by $t \gg \tau=1 /$ $\left(4 \pi a^{2} n * v\right)$. Using values for the escape flux $(n * v>4 \times$ $10^{11} \mathrm{~cm}^{-2} \mathrm{~s}^{-1}$ ) from section 4 and ice particle sizes $a>5 \mu \mathrm{m}$, we find that $\tau$ is many orders of magnitude smaller than the timescales of typically minutes over which ambient temperature, trace gas partial pressure, and $\mathrm{H}_{2} \mathrm{O}$ deposition rate (hence $a$ ) change. Therefore the steady state assumption is safe in the case of $\mathrm{HNO}_{3}$ and presumably for most other trace gases; smaller escape fluxes that would lead to larger $\tau$ favor the burial regime and lessen the role of surface kinetics during trapping.

\section{Comparison With Observations}

[31] The aim of this section is to constrain the two open parameters of the updated trapping model, $v$ and $n_{*}$, by comparison to available laboratory and field data of the uptake of $\mathrm{HNO}_{3}$ by growing ice surfaces in section 4.1 and section 4.2, respectively. Both types of measurements cannot distinguish between surface-adsorbed $\mathrm{HNO}_{3}$ or $\mathrm{HNO}_{3}$ trapped in the bulk ice. A unique set of values for $v$ and $n_{*}$ is inferred from both data sets and enables the validation of the model for this important atmospheric trace 
gas by explaining the observed trends of uptake as a function of temperature, ice growth rate, and partial pressure.

[32] Although $\mathrm{HNO}_{3}$ is known to dissociate upon adsorption, the use of the nondissociative Langmuir isotherm equation (4a) appears to be a fair approximation, given the relatively small differences between dissociative and nondissociative isotherms and the uncertainties in experi-
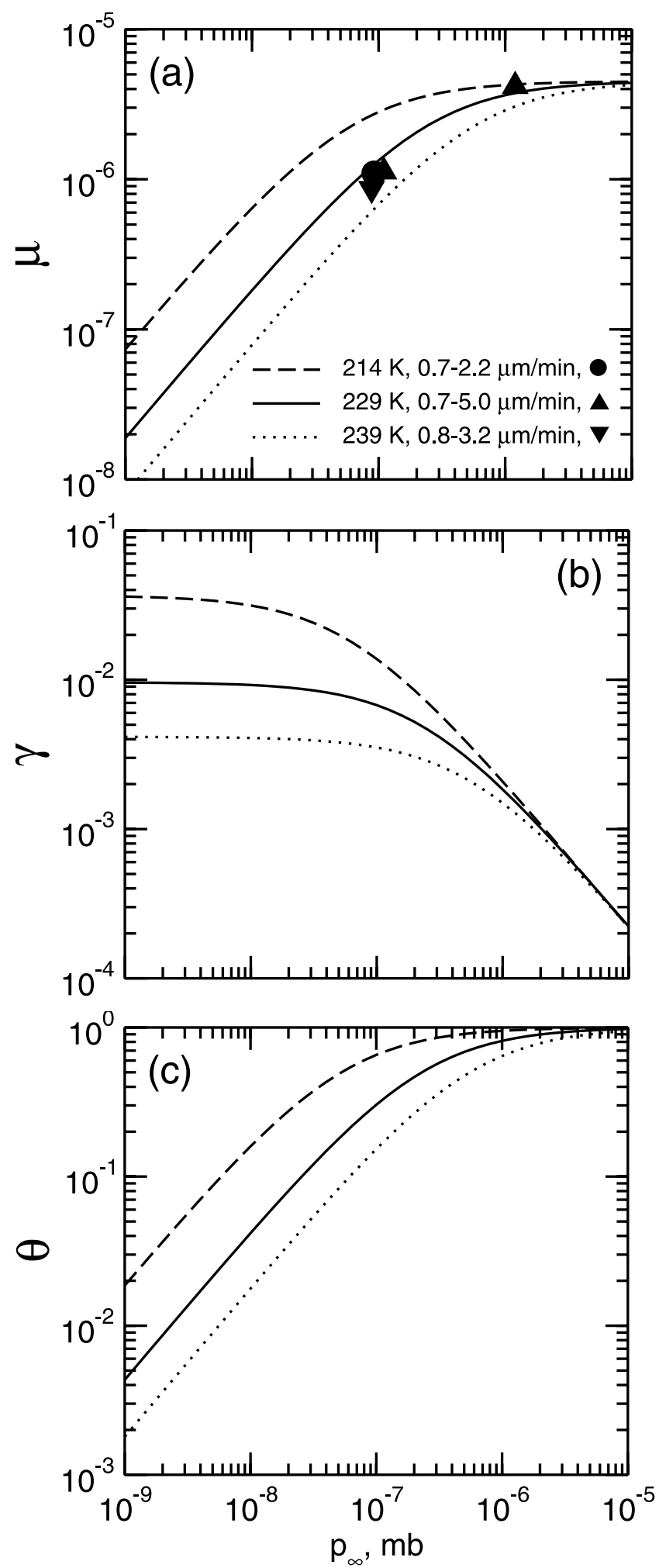

mentally determining the equilibrium constants [Ullerstam et al., 2005].

\subsection{Laboratory Data}

[33] Laboratory measurements of $\mathrm{HNO}_{3}$ uptake by growing ice surfaces in upper tropospheric conditions have been discussed within the Atmospheric Chemistry community [Heard, 2005] and first experimental results have been reported by Ullerstam and Abbatt [2005]. Those experiments were carried out using an ice coated-wall flow tube coupled to a chemical ionization mass spectrometer (CIMS). The ice surfaces were prepared as smooth (at the molecular level) ice films by rapid freezing of ultrapure water. (See Abbatt et al. [2008] for an experimental validation of this assumption.) Supersaturation with respect to the ice phase was achieved by increasing the relative humidity of the carrier gas entering the flow tube, triggering ice growth. The CIMS measurements determine the amount of $\mathrm{HNO}_{3}$ lost in the gas phase due to uptake in the growing ice film. The ice content added during growth is determined from the average supersaturation and the distance over which the new ice film forms. Therefore the inferred molar ratios are not sensitive to the amount of ice present prior to growth.

[34] The results of these measurements are presented in Figure $3 \mathrm{a}$ (symbols) as molar ratios $\mu$ versus ambient partial pressure $p_{\infty}$. Temperatures $T$ and ranges of ice growth rates $\dot{x}$ realized during the experiments are given in the legend and correspond to those typically found in midlatitude cirrus clouds. The data reveal four distinct features. (1) $\mu$ does not increase in proportion to the partial pressure; a number of measurements at each $p_{\infty}$ and sufficiently low overall uncertainties exclude a linear dependence. (2) There is little, if any, dependence of $\mu$ on $\dot{x}$. (3) There appears to be only a small dependence on $T$. (4) The $\mathrm{HNO}_{3}$ uptake in the presence of growing ice surfaces is substantially higher than at full thermodynamic equilibrium that establishes over long timescales by diffusion and solubility of $\mathrm{HNO}_{3}$ in bulk ice.

[35] Concerning issue (4), Ullerstam and Abbatt [2005] have already remarked that the $\mathrm{HNO}_{3}$ solubility limits inferred from laboratory data [Thibert and Dominé, 1998] predict substantially smaller $\mu$ values than measured. This clearly demonstrates that processes other than those controlling a solid solution of $\mathrm{HNO}_{3}$ in ice played an important role in these nonequilibrium experiments.

[36] We analyze the issues (1)-(3) in more detail with the help of the trapping model. The curves in Figure $3 \mathrm{a}$ are the

Figure 3. (a) Molar ratios $\mu$ of $\mathrm{HNO}_{3}$ in ice versus $\mathrm{HNO}_{3}$ partial pressure $p_{\infty}$ for different temperatures $T$ and ice growth rates $\dot{x}$ (legend). Solid symbols represent laboratory measurements [Ullerstam and Abbatt, 2005]; combined errors are below $\sim 30 \%$. Curves are trapping model results in the free molecular flow regime using the measured Langmuir equilibrium constant. All curves are predicted close to the adsorption limit, where the dependence of $\mu$ on $\dot{x}$ is very small, and have therefore been computed for an average $\dot{x}=2 \mu \mathrm{m} / \mathrm{min}$. (b) Modeled uptake coefficients $\gamma$ and (c) fractional coverages $\theta$ consistent with the trapping solution. 
model results obtained in the free molecular flow regime (section 3.2), although gas diffusion probably played some role in limiting uptake in the experiments. To constrain the unknown parameters, we have used the Langmuir constant recommended by the International Union for Pure and Applied Chemistry (IUPAC) [Ammann et al., 2008]

$$
K_{L}\left[\mathrm{mb}^{-1}\right]=\frac{2}{T[\mathrm{~K}]} \exp \left(\frac{4585}{T[\mathrm{~K}]}\right)
$$

which is consistent with data taken with the same experimental setup as used in the nonequilibrium experiments discussed here but using static ice surfaces [Ullerstam et al., 2005; Cox et al., 2005]. Equation (20a) returns $K_{L}$ in units of $\mathrm{mb}^{-1}$ when $T$ is inserted in units of $\mathrm{K}$; the associated adsorption enthalpy is given by $k_{B} 4585 \mathrm{~K} \simeq 9 \mathrm{kcal} / \mathrm{mol}$.

[37] A recent analysis has shown that besides a conventional desorption rate $k_{L}$, associated with $K_{L}$ via equation (5b), diffusive loss of adsorbed $\mathrm{HNO}_{3}$ molecules into the ice film needs to be invoked to explain the static ice data [Cox et al., 2005], reducing the effect of desorption and resulting in a net escape rate $k$. The corresponding overall equilibrium constant $K$ that provides the link to the escape speed $v$ and to $n *$ via equation (5a) is given by $K\left[\mathrm{mb}^{-1}\right]=$ $(12.3 \pm 0.9) \times 10^{7}-(5.1 \pm 0.4) \times 10^{5} T[\mathrm{~K}]$ [Ullerstam et $a l ., 2005]$. Strictly speaking, we must use $K$ in the trapping model. In practice, however, the difference between $K$ and $K_{L}$ is small and we opt to employ $K_{L}$ because of its van't Hoff-type temperature dependence.

[38] This being said, we compute $v$ from equation (5a) by using equation (20a), yielding

$$
v\left[\frac{\mu \mathrm{m}}{\min }\right]=2 \times 10^{9} \sqrt{T[\mathrm{~K}]} \exp \left(-\frac{4585}{T[\mathrm{~K}]}\right)
$$

To arrive at this expression, we have used for $\mathrm{HNO}_{3}$ the deposition coefficient on ice $\alpha=0.3$ [Hanson, 1992; Abbatt, 1997], the thermal speed $u[\mathrm{~cm} / \mathrm{s}] \simeq 1832 \sqrt{T[\mathrm{~K}]}$, and $n_{*}=$ $1.5 \times 10^{17} \mathrm{~cm}^{-3}$. Along with the experimental range of $T$ and $\dot{x}$, these parameters predict that trapping takes place near the adsorption limit (section 3.4), i.e., $\mu \approx \mu_{\text {ads }}=$ $\nu_{w} n_{*} \theta$. Molar ratios scale linearly with $p_{\infty}$ below $p_{\infty} \simeq$ $10^{-7} \mathrm{mb}$ in the unsaturated surface adsorption regime and level off above, approaching a common value $\nu_{w} n_{*} \simeq 4.5 \times$ $10^{-6}$. Furthermore, molar ratios decrease with increasing $T$, most pronounced at low $p_{\infty}$.

[39] As expected for a solution close to the adsorption limit, the trapping model does not predict any significant dependence of $\mu$ on $\dot{x}$ (issue ii) within the parameter ranges indicated in the legend, consistent with the data taken by Ullerstam and Abbatt [2005]. This is fortunate, as the experimentally determined growth rates must be regarded as rough estimates due to the inhomogeneity of supersaturation across the ice sample area. We have therefore computed all curves in Figures $3 \mathrm{a}$ and $3 \mathrm{~b}$ for an average ice growth rate of $\dot{x}=2 \mu \mathrm{m} / \mathrm{min}$.

[40] The modeled $T$ dependence of $\mu$ (issue (3)) is directly tied to the prescribed equilibrium constant via $v$. The model predicts a moderate temperature variation of $\mu$ for medium partial pressures, because of the transition to the surface-saturated adsorption limit. The latter explains why the $p_{\infty}$ dependence is not linear (issue (1)). The $T$ variation seems to be more pronounced than suggested by the data near $p_{\infty}=10^{-7} \mathrm{mb}$. It would be desirable to perform further experiments at lower $p_{\infty}$, where $\mu$ is expected to be more sensitive to variations in $T$, to help resolve this issue.

[41] The above comparison builds further confidence in the usefulness of the trapping concept to explain the basic features controlling the uptake of $\mathrm{HNO}_{3}$. Therefore we present supporting information from the trapping model in Figure $3 \mathrm{~b}$, showing the uptake coefficient $\gamma$ versus $p_{\infty}$. The fact that $\gamma$ values fall off rapidly for $p_{\infty}>10^{-7} \mathrm{mb}$ indicates that uptake becomes suppressed as soon as the surface becomes saturated. Importantly, $\gamma$ values are smaller than $\alpha$ by at least one order of magnitude, implying that the escape speed exceeds the ice growth rate. In fact, equation (20b) predicts $v \simeq 15-144 \mu \mathrm{m} / \mathrm{min}$ for $T=214-239 \mathrm{~K}$, well above the estimated ice growth rates $\dot{x}=0.7-5 \mu \mathrm{m} / \mathrm{min}$, so that at low $p_{\infty}, \gamma$ decreases with increasing $v$ (increasing $T$ ), see Table 2. Because the adsorption limit is approximately realized in this experiment, it is meaningful to present the fractional coverages $\theta$ (Figure $3 \mathrm{c}$ ) associated with $\mu_{\text {ads. }}$. The coverages exhibit saturation with rising $p_{\infty}$, reflecting the isotherm equation (19a).

\subsection{Field Observations}

[42] In analyzing aircraft field observations, we focus on the dependence of trapping on the trace gas partial pressure. The general problem that arises in such an exercise is that $p_{\infty}$ varies greatly because of spatial inhomogeneities caused by transport and chemistry, including the trapping process. Also, sedimenting ice particles may experience different $p_{\infty}$ values during their lifetime. (For these reasons, we do not consider local depletion of the gas phase by trapping in this analysis.) Furthermore, average $p_{\infty}$ values used to estimate the trapped amount may not be good indicators of the actual values present during ice growth. As $T$ varies much less than $p_{\infty}$, a comparison of trapped molar ratios as a function of $T$ like in KV06 is more robust. In that study, limitations in the interpretation of airborne measurements have been discussed and approximate expressions for the mean IWC $(T)$ and mean radius $a(T)$ of ice particles have been presented. The latter relationships are also employed here to evaluate the ice growth equation (1).

[43] Two observational studies investigated uptake in a wide range of $\mathrm{HNO}_{3}$ partial pressures, the Cirrus Regional Study of Tropical Anvils and Cirrus Layers-Florida Area Cirrus Experiment (CRYSTAL-FACE [Popp et al., 2004]) and the European Polar Stratospheric Cloud and Leewave Experiment (EUPLEX [Voigt et al., 2006]). The former study measured $\mathrm{HNO}_{3}$ directly mostly in convectively generated cold cirrus using an aircraft CIMS instrument. The latter study detected total reactive nitrogen $\left(\mathrm{NO}_{y}\right.$, of which $\mathrm{HNO}_{3}$ is only a part) in Arctic cirrus by chemically reducing $\mathrm{NO}_{y}$ to nitrogen oxide (NO) and detecting $\mathrm{NO}$ through chemiluminescence from its reaction with ozone. These two aircraft measurements provide us with a sufficient number of data points with variable $p_{\infty}$ in a sufficiently narrow range of $T$ to enable an analysis of trapping of $\mathrm{HNO}_{3}$ as a function of its partial pressure.

[44] The top panel in Figure 4 depicts the field data as a function of the $\mathrm{HNO}_{3}$ partial pressure $p_{\infty}$ for the two campaigns (circles with dashed lines). The CRYSTAL- 

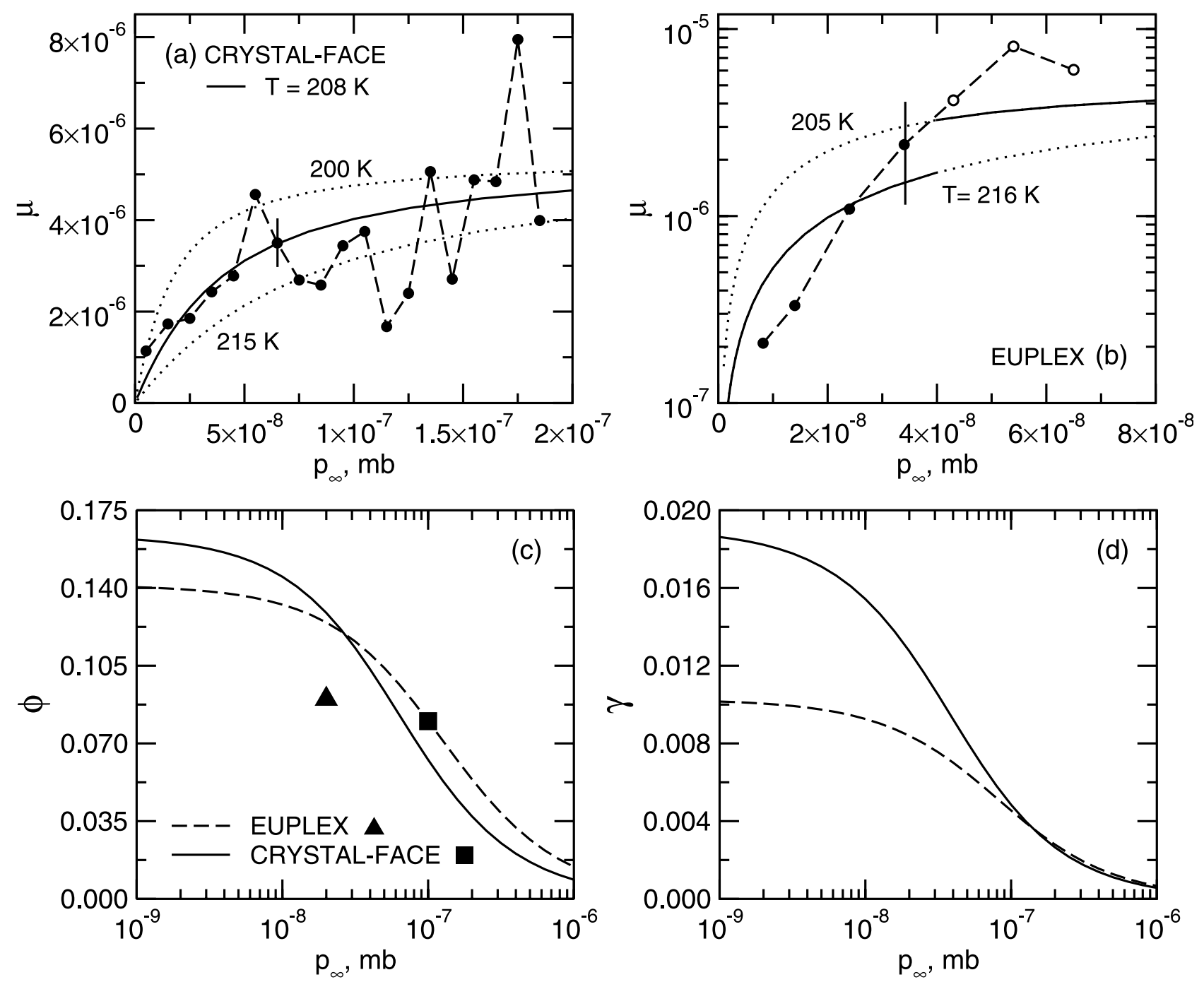

Figure 4. Molar ratios $\mu$ of $\mathrm{HNO}_{3}$ in ice versus $\mathrm{HNO}_{3}$ partial pressure $p_{\infty}$ (curves) evaluated from the general trapping solution. Circles are results from (a) CRYSTAL-FACE [Popp et al., 2004] and (b) EUPLEX [Voigt et al., 2006] airborne campaigns. Corresponding average air temperatures are indicated. A representative error bar obtained from a propagation of the uncertainties of the $\mathrm{HNO}_{3}\left(\mathrm{NO}_{y}\right)$ and water measurements is given for each campaign. Most of the EUPLEX data have been obtained around $p_{\infty}=2 \times 10^{-8} \mathrm{mb}$ (solid circles); the data statistic is poor at higher values in significantly colder conditions (open circles). Trapping model results for (c) the ice phase fraction $\phi$ and (d) the uptake coefficient $\gamma$ over a wide range of $p_{\infty}$ in CRYSTAL-FACE (solid curves) and EUPLEX (dashed) conditions. Symbols indicate mean values estimated from the data; associated variabilities are much larger than the mean.

FACE measurements (campaign average $T=208 \mathrm{~K}$ and $P=170 \mathrm{mb}$ ) cover the entire range of shown $p_{\infty}$ values with equally high data density (Figure 4a). The EUPLEX data statistic is generally poorer, and only $2 \%$ of all data points have been taken above $4 \times 10^{-8} \mathrm{mb}$, as indicated by open circles (Figure 4b). Data points plotted as filled (open) circles are associated with the campaign average $T=216 \mathrm{~K}$ $(T=205 \mathrm{~K})$. The average air pressure during EUPLEX was $P=200 \mathrm{mb}$.

[45] The solid curves are trapping model results from equation (9a) using equation (1) evaluated at the campaign average $T$ and $P$, IWC related to supersaturation and hence $\dot{a}$ as in equation (15b) with $z=1, v(T)$ from equation (20b), and $n_{*}=1.8 \times 10^{17} \mathrm{~cm}^{-3}$. We stress that these choices for $v$ and $n *$ are consistent with those from the laboratory analysis (section 4.1). Constrained in this way, our updated model provides an excellent representation of the averaged airborne data from CRYSTAL-FACE. Much of the scatter around the solid curve can be explained by lower and higher $T$ or IWC (dotted curves). We also regard the comparison with the EUPLEX measurements as good, as those have only been empirically corrected for the fraction of $\mathrm{HNO}_{3}$ contained in measured $\mathrm{NO}_{y}$. The apparent $T$ dependence of the molar ratios (solid versus open circles) is roughly captured by the model.

[46] The bottom panel in Figure 4 provides additional information from the trapping model, namely the average fraction of $\mathrm{HNO}_{3}$ in ice, $\phi=1-\phi_{\infty}$ (Figure 4c), and the 
uptake coefficient for trapping $\gamma$ (Figure 4d). Both quantities decrease with increasing $p_{\infty}$ as the uptake becomes increasingly suppressed by surface saturation. In the limit of unsaturated surface adsorption, realized for $p_{\infty} \simeq 10^{-9} \mathrm{mb}$ for both CRYSTAL-FACE (solid curves) and EUPLEX (dashed), the $\gamma$ values (0.01 and 0.018) are much smaller than the accommodation coefficient $\alpha=0.3$ (Figure $4 \mathrm{~d}$ ). This indicates that most efficient trapping (burial, for which $\gamma \rightarrow \alpha$ ) was not realized given the mean temperatures
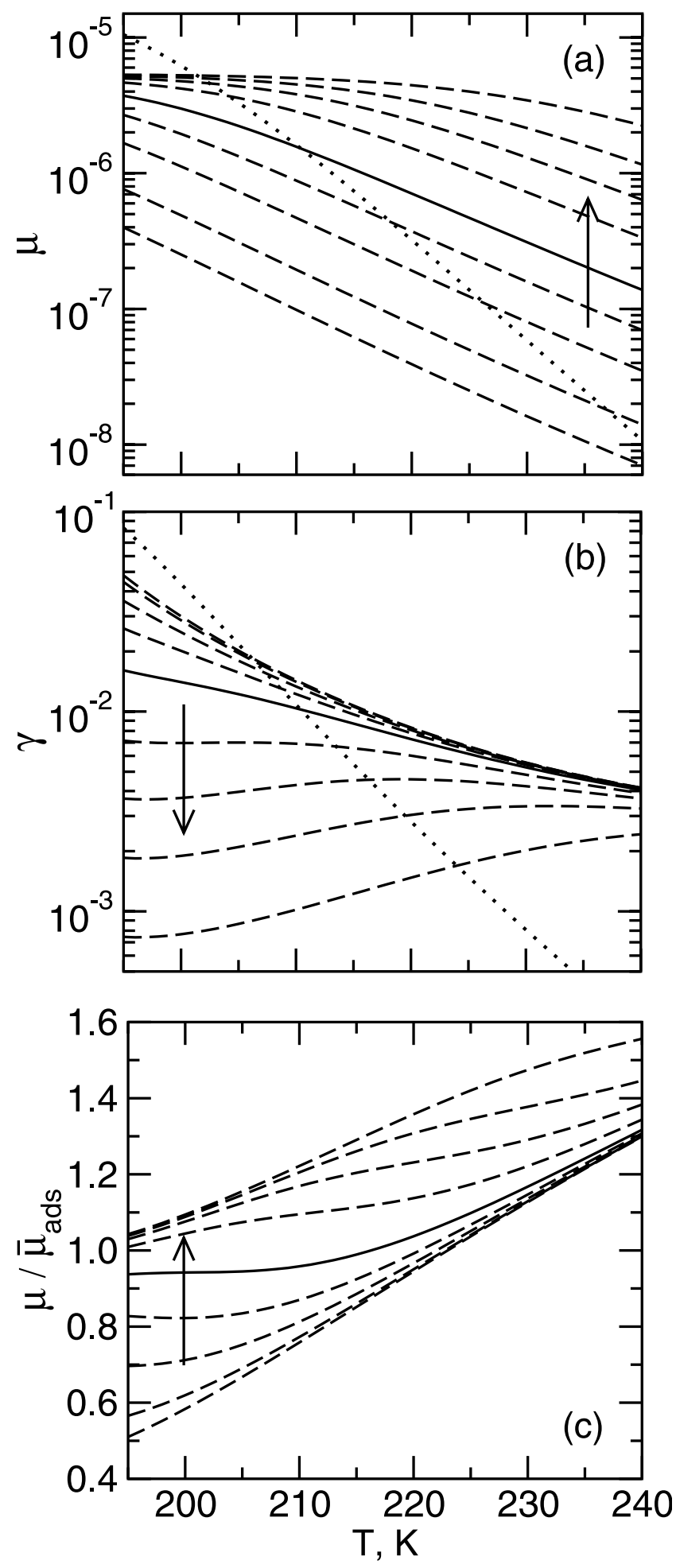

( $208 \mathrm{~K}$ and $216 \mathrm{~K})$. This point will be reiterated in section 5.1. We estimate the average ice particle growth rates prevalent during the two airborne measurements to be $\dot{a} \simeq 0.5 \mu \mathrm{m} / \mathrm{min}$. The corresponding escape speeds are in the range $v \simeq 8-18 \mu \mathrm{m} / \mathrm{min}$. This finding is consistent with the result from $\mathrm{KV06}$, indicating that the burial regime for $\mathrm{HNO}_{3}$ in cirrus clouds (in which $\dot{a}>v$, or more precisely, $\xi \ll 1$ and $\zeta \ll 1$ ) is not realized above $\sim 203 \mathrm{~K}$ at $p_{\infty}=2 \times$ $10^{-8} \mathrm{mb}$.

[47] For the mean $p_{\infty} \simeq 10^{-7} \mathrm{mb}\left(2 \times 10^{-8} \mathrm{mb}\right)$ measured during CRYSTAL-FACE (EUPLEX), an average $\phi \simeq 6 \%(12 \%)$ of the total number of $\mathrm{HNO}_{3}$ molecules partitions in the cirrus ice phase (Figure 4c). Values $\phi$ depend on the actual cirrus ice water content and $\mathrm{HNO}_{3}$ partial pressure, which are highly variable. Indeed, a large range of partitioning fractions have been found during CRYSTAL-FACE, however, at the mean IWC $=2 \mathrm{mg} / \mathrm{m}^{3}$ used in the trapping model, observed $\phi \simeq 8 \%$ inferred using Figures 9 and 13 from Popp et al. [2004] is consistent with our estimate within the measurement uncertainty. The average ice phase fraction of $\sim 9 \%$ at a slightly larger average IWC estimated from EUPLEX data [Voigt et al., 2006] is also close to our model estimate.

\section{Discussion}

[48] In this section, we will discuss the trapping results over the wide temperature and partial pressure ranges where $\mathrm{HNO}_{3}$ uptake in cirrus has been observed in field experiments. Furthermore, we will compare our updated trapping model with the previous version without surface saturation effects. Discussing two recent data sets taken at very low temperatures helps identify open issues in understanding the field data (section 5.1).

[49] The updated trapping model does not explicitly resolve molecular processes that occur in the surface layer of the ice. While this simplification appears to be justified for $\mathrm{HNO}_{3}$ according to our results from section 4, resolving the surface layer may become necessary in other cases. We address this issue by outlining the necessary modifications to our methodology (section 5.2).

\subsection{Model Evaluation}

[50] The main results of the updated trapping model useful for comparison with atmospheric studies are shown in Figure 5. The molar ratios $\mu$ and uptake coefficients $\gamma$ have been obtained from combining equations (9a), (12),

Figure 5. Trapping model results for $\mathrm{HNO}_{3}$ in upper tropospheric/lower stratospheric conditions for (a) molar ratio $\mu$, (b) uptake coefficient $\gamma$, and (c) ratio of $\mu$ that is trapped versus bulk $\bar{\mu}_{\text {ads }}$ that would be adsorbed at zero ice growth rate. The variables are shown as a function of air temperature $T$ and $\mathrm{HNO}_{3}$ partial pressures $p_{\infty}=10^{-9}, 2 \times$ $10^{-9}, 5 \times 10^{-9}, 10^{-8}, 2 \times 10^{-8}, 5 \times 10^{-8}, 10^{-7}, 2 \times 10^{-7}$, $5 \times 10^{-7} \mathrm{mb}$. Results for $p_{\infty}=2 \times 10^{-8} \mathrm{mb}$ are plotted as solid curves. Arrows indicate the direction of increasing $p_{\infty}$. Dotted curves in Figures $5 \mathrm{a}$ and $5 \mathrm{~b}$ result from a fit of the previous trapping model neglecting surface-saturated adsorption to field data assuming the same fixed value for $p_{\infty}$ as the solid curves. 
and (13). Both variables have been evaluated at $P=200 \mathrm{mb}$ over the range of temperatures $T=195-240 \mathrm{~K}$ and $\mathrm{HNO}_{3}$ partial pressures $p_{\infty}=10^{-9}-5 \times 10^{-7} \mathrm{mb}$ observed in the upper troposphere and lower stratosphere region (dashed curves). The approximate expressions used to estimate typical average values for the mean ice particle radius and ice water content, taken from KV06, are valid within the stated range of $T$.

[51] Below $p_{\infty} \simeq 2 \times 10^{-8} \mathrm{mb}$ (solid curves) at all $T$, trapping increases in importance as $T$ decreases, corroborating our previous finding based on the analyses of atmospheric data from a large number of field campaigns [KV06]. In this region, both the molar ratios (Figure 5a) and the uptake coefficients (Figure 5b) increase toward colder $T$, approaching but not reaching the burial limit (where $\gamma \rightarrow \alpha=$ 0.3 ). This trend is reversed for $\gamma$ above $p_{\infty} \simeq 2 \times 10^{-8} \mathrm{mb}$, because adsorption saturates as higher partial pressures and particularly at $T<220 \mathrm{~K}$, where the escape rates become smaller and molecules are more likely to stay adsorbed. Consequently, the rate of uptake is reduced and $\mu$ increases much weaker or even levels off at the adsorption limit $\mu \simeq$ $5 \times 10^{-6}$.

[52] In sum, we find that $\mathrm{HNO}_{3}$ uptake in cirrus ice particles is determined by both adsorption and diffusional growth. The latter plays a significant role only at low $(T<$ $210 \mathrm{~K})$ temperatures and low $\left(p_{\infty}<2 \times 10^{-8} \mathrm{mb}\right) \mathrm{HNO}_{3}$ partial pressures. If $p_{\infty}$ increases at low $T$, surface-saturated adsorption limits the trapping efficiency. If $T$ increases, regardless of $p_{\infty}$, escape speeds much higher than growth rates lead to small trapping efficiencies. A key finding of this study is that $\mathrm{HNO}_{3}$ uptake by trapping does not result in molar ratios exceeding $\sim 5 \times 10^{-6}$.

[53] It is instructive to compare trapped molar ratios $\mu$ with values that would result from interaction with a nongrowing ice particle in otherwise unchanged conditions. We recall that in this case, the respective molar ratio $\mu_{\mathrm{ads}}$ from equation (19b) relates to the surface layer only (section 3.4), i.e., it is defined as the ratio of $\mathrm{HNO}_{3}$ molecules, $N$, to the number of $\mathrm{H}_{2} \mathrm{O}$ molecules, $N_{w, l}=4 \pi\left[(a+\delta)^{3}-a^{3}\right] /$ $\left(3 \nu_{w}\right)$, in the layer with a characteristic depth $\delta$. The remaining core volume is $\mathrm{HNO}_{3}$ free. To obtain values for adsorbed molar ratios that are representative for the entire ice particle and thus directly comparable to $\mu$, we correct $\mu_{\text {ads }}$ by adding the number of $\mathrm{H}_{2} \mathrm{O}$ molecules present in the core, $N_{w}=$ $4 \pi a^{3} /\left(3 \nu_{w}\right)$, to $N_{w, l}$. This results in modified adsorbed molar ratios, $\bar{\mu}_{\mathrm{ads}}=N /\left(N_{w, l}+N_{w}\right)=\mu_{\mathrm{ads}} /(1+y) \leq \mu_{\mathrm{ads}}$, with the correction factor $1 / y=(1+\delta / a)^{3}-1$. The depth parameter is estimated by $\delta=1 /\left(\sigma n_{*}\right) \simeq 13 \mu \mathrm{m}$ (section 2$)$, using $\sigma=4.2 \times$ $10^{-15} \mathrm{~cm}^{2}$ for $\mathrm{HNO}_{3}$ on ice.

[54] The ratio $\mu / \bar{\mu}_{\text {ads }}$ is shown in Figure 5c. At high $T$ (in the upper right quarter of Figure 5), the ratio exceeds unity because the ice particles are larger than the characteristic layer depth $(a(T)>\delta)$, so that $\bar{\mu}_{\text {ads }}$ becomes small due to additional dilution by bulk phase $\mathrm{H}_{2} \mathrm{O}$ molecules. At low $T$ (in the lower left quarter), we have $a(T)<\delta$ so that the dilution effect diminishes and $\bar{\mu}_{\text {ads }} \approx \mu_{\text {ads }}$. Here the ratio $\mu / \bar{\mu}_{\text {ads }}$ falls below unity as we leave the surface-saturated regime with decreasing $p_{\infty}$. This discussion emphasizes that the application of static adsorption isotherms in explaining field observations in cirrus conditions may lead to systematic errors of up to a factor of two in quantifying the molar ratio of ice-bound vapor.
[55] The CRYSTAL-FACE analysis resulted in a mean number of $\mathrm{HNO}_{3}$ molecules per unit ice particle surface area of $N_{s}=2 \times 10^{13} \mathrm{~cm}^{-2}$ at $T=208 \mathrm{~K}$ and $p_{\infty} \simeq 10^{-7} \mathrm{mb}$, assuming that the measured $\mathrm{HNO}_{3}$ resided in the surface layers of the cirrus ice particles. We transform this value into a bulk molar ratio equivalent to $\bar{\mu}_{\text {ads }}$. The number of adsorbed trace gas molecules in ice particles with an average radius $a$ is given by $N=N_{s} \cdot 4 \pi a^{2}$. The number of $\mathrm{H}_{2} \mathrm{O}$ molecules contained in the ice particle including the surface layer is $N_{w}=4 \pi a^{3} /\left(3 \nu_{w}\right)$. Hence $\bar{\mu}_{\text {ads }}=N_{s} \cdot 3 \nu_{w} / a$, decreasing with increasing particle size. Inserting the observed surface coverage and our estimate for the average ice particle radius, $a(T) \simeq 16 \mu \mathrm{m}$, we find $\bar{\mu}_{\text {ads }} \simeq 10^{-6}$, which is significantly below the values $\sim 4 \times 10^{-6}$ measured at the $T$ and $p_{\infty}$ values given above (Figure $4 \mathrm{a}$ ). As most clouds had volume mean radii larger than $10 \mu \mathrm{m}$ [Popp et al., 2004], our estimate for $\bar{\mu}_{\text {ads }}$ may represent an upper limit.

[56] We now compare our findings with those from the previous version of the trapping model that used an unsaturated isotherm [KB04]. This previous version had been used to constrain the trapping efficiency $\epsilon$ by an eyeball fit to field measurements [KV06]. (Essentially $\xi=v / \dot{a}$ was fitted; the parameter $n_{*}$ did not enter that model because adsorption was generally assumed to be unsaturated.) The corresponding results from that study are shown as dotted curves in Figures $5 \mathrm{a}$ and $5 \mathrm{~b}$. The dotted and solid curves have been calculated for $p_{\infty}=2 \times 10^{-8} \mathrm{mb}$ and can therefore be directly compared. The dotted curves cut across a wide range of $\mu$ and $\gamma$ curves, because the underlying fit did not consider the true partial pressure dependence of the field data which were taken in a range of $p_{\infty}$ conditions. This underscores the importance of considering effects of variability in $p_{\infty}$ in determining the true $\mu$ and $\gamma$ values. The updated model provides a physically based description of the partial pressure dependence of trapping fully constrained by available laboratory information, replacing the empirical assumptions in our previous study [KV06].

[57] We describe two additional aircraft data sets that hint at very efficient $\mathrm{HNO}_{3}$ uptake at very low mean air temperatures $(187 \mathrm{~K})$ and air pressures $(80-100 \mathrm{mb})$. The first describes CIMS measurements during the Costa Rica Aura Validation Experiment (CR-AVE) [Popp et al., 2007] in a subvisible cirrus cloud at $183-191 \mathrm{~K}$, with a mean value of $\mu=1.4 \times 10^{-5}$ attained at low $p_{\infty} \simeq 2.5 \times 10^{-9} \mathrm{mb}$. The second was taken during the Stratospheric Climate Links with Emphasis on the Upper Troposphere and Lower Stratosphere (SCOUT) project's tropical campaign at $185-191 \mathrm{~K}$, revealing a mean $\mu=4.7 \times 10^{-5}$ attained at $p_{\infty} \simeq 3 \times 10^{-8} \mathrm{mb}$. The updated trapping model, when applied in these conditions with IWC and $a$ from the measurements, is not consistent with the CR-AVE and SCOUT data. It underestimates the observed $\mu$ values by up to a factor of 10 .

[58] We offer a mechanism that is in principle capable of explaining this discrepancy. We postulate that $\mathrm{HNO}_{3}$ is present in the freezing aerosol particles and a fraction may be retained in the ice particles after freezing and depositional growth [KV06]. If we make the plausible assumption that the subset of freezing particles is mainly composed of supercooled aqueous sulfuric acid, a large amount of $\mathrm{HNO}_{3}$ will dissolve below $190 \mathrm{~K}$ in the liquid solution at the prevailing $\mathrm{HNO}_{3}$ concentrations. (The 
amount of $\mathrm{HNO}_{3}$ dissolved in liquid particles prior to freezing is small compared to the trapped amount for $T>$ $195 \mathrm{~K}$ [KV06].) In this case, it is possible that the amount of ice-bound $\mathrm{HNO}_{3}$ originating from the freezing process exceeds that of trapped $\mathrm{HNO}_{3}$, if the ice particles stay relatively small on average. Microphysical simulations have shown that $\mu$ values exceeding $10^{-5}$ can be generated in this way in sufficiently small ice crystals [Voigt et al., 2007, their Figure 8].

\subsection{Resolved Ice Surface Layer}

[59] The introduction of a velocity or rate of escape from an unresolved surface layer (Figure 1a) served to minimize the number of free parameters in describing the trapping in growing ice surfaces or particles. In view of the sparsity of experimental data available to constrain any theory of uptake, this approach appears to be justified. Nevertheless, the question arises under which circumstances this simplification might fail to predict details of the uptake process.

[60] To answer this question, we generalize the trapping concept (Figure 1b). Adsorbed molecules now enter the surface layer that is associated with the true thickness $\delta$. Instead of merging potential kinetic processes in the layer into a net escape speed, we now consider explicitly the desorption rate $k_{L}$ to account for reversible adsorption, as well as forward (surface $\rightarrow$ bulk), $R_{s-}$, and backward (bulk $\rightarrow$ surface), $R_{-s}$, rates that compete with desorption and couple the surface layer to the bulk phase. The latter rates $R$ may be associated with diffusion through the surface layer into pores and along grain boundaries in the ice.

[61] To simplify matters, we restrict our analysis to planar (or free molecular) flow. The fluxes at the gas-surface and surface-bulk interfaces are now given by

$$
\begin{gathered}
f_{+}=-\left(1-n_{s} \delta \sigma\right) n_{\infty} \alpha u / 4+k_{L} \delta n_{s}, \\
f_{s}=-\left(R_{s-} \delta+\dot{x}\right) n_{s}+R_{-s} \delta n_{-}, \\
f_{-}=-n_{-} \dot{x} .
\end{gathered}
$$

Setting $f_{+}=f_{s}=f_{-}$yields two equations for the two unknowns $n_{-}$and $n_{s}$; the latter is the number concentration of molecules in the surface layer that is limited by the maximum possible concentration $1 /(\delta \sigma)$. In this model, $k_{L}$ (following from $K_{L}$ ) and $n_{s}$ (determined by the above flux equations) are no open parameters. Instead, $\delta, R_{s-}$, and $R_{-s}$ are three open parameters.

[62] After conversion to our previous notation, we obtain the solution in terms of $\mu$ and $\gamma$ :

$$
\begin{gathered}
\mu=\nu_{w} n_{\infty} \frac{\kappa^{\prime}}{1+\kappa^{\prime}(\lambda+\vartheta) \mathcal{C}}, \quad \gamma=\alpha \frac{1}{1+\kappa^{\prime}(\lambda+\vartheta) \mathcal{C}} \\
\mathcal{C}=\frac{\dot{x}+R_{-s} \delta}{\dot{x}+R_{s-} \delta}
\end{gathered}
$$

These results are identical to those from section 3.2, except for the factor $\mathcal{C}$. We recover our original results for $\mathcal{C}=1$. This implies that our simplified approach describes trapping well either if $R_{-s}=R_{s-}$ (forward and backward rates at the surface-bulk interface are of similar magnitude) or if both $R_{-s} \delta \ll \dot{x}$ and $R_{s-} \delta \ll \dot{x}$ (rates do not matter because the rate of material growth is much larger).

[63] We revisit the analysis of the uptake data from section 4.1 which have been fairly well explained using the updated trapping model $(\mathcal{C}=1)$. Cox et al. [2005] have shown that diffusion into surface layers in the ice occurred during the laboratory experiments, i.e., $R_{s_{-}}>0$. They roughly estimate a diffusion coefficient $D=10^{-12} \mathrm{~cm}^{2} / \mathrm{s}$ independent of $T$ and a diffusion depth $\delta=0.06-1 \mu \mathrm{m}$. These numbers translate into a "diffusion velocity" $v_{D}=$ $R_{s-} \delta=D / \delta=0.006-0.12 \mu \mathrm{m} / \mathrm{min}$ to be compared with the ice film growth rates $\dot{x}=0.7-5 \mu \mathrm{m} / \mathrm{min}$ in the corresponding trapping experiments. Hence we conclude that $v_{D} \ll \dot{x}$, which in turn implies $\mathcal{C} \simeq 1$ if we assume that $R_{-S} \delta \ll \dot{x}$ as well.

[64] The generalization of equations (21a) and (21b) to spherical diffusional flow and atmospheric ice particles is straightforward and can be established if needed for specific trace gases. To apply this expanded model, detailed measurements are required to constrain the parameters affecting $\mathcal{C}$. The latter are difficult to obtain from atmospheric studies, stressing the role of detailed laboratory experiments.

\section{Conclusions}

[65] We have generalized our previous trapping theory to include the effect of surface-saturated adsorption, which acts to limit the uptake if the trace gas partial pressures become sufficiently high. The model provides the variables requried to calculate the trapping rate as a function of ambient conditions. Trapped amounts have been discussed in terms of steady state bulk phase molar ratios in ice. We have shown that the trapping model reduces to the underlying adsorption equilibrium model in the limit of nongrowing particles and expresses the fractional surface coverage in terms of a molar ratio of trace gas molecules in the adsorption layer. The use of an equilibrium surface coverage model is not appropriate for ice crystals that are subject to net depositional growth.

[66] We have applied the updated model to observations of $\mathrm{HNO}_{3}$ uptake in a wide range of partial pressures, ice growth rates, and temperatures, thereby achieving consistency between laboratory and field data. The open model parameters, escape speed and maximum trapped concentration of $\mathrm{HNO}_{3}$ molecules, have been determined consistently from both data sets, the key features of which could be reproduced fairly well. In the meteorological and chemical conditions prevalent during the field measurements, $\mathrm{HNO}_{3}$ uptake was neither controlled purely by adsorption nor purely by diffusion (burial). This highlights the importance of using an uptake model that connects both adsorption and burial regimes, as well as surface-saturated and unsaturated regimes, to interpret atmospheric observations.

[67] The trapping mechanism is required to explain most of the $\mathrm{HNO}_{3}$ observations. The highest molar ratio in cirrus ice particles is predicted to be $\sim 5 \times 10^{-6}$, attained at low air temperatures $(<210 \mathrm{~K})$ and high partial pressures $(>2 \times$ $10^{-7} \mathrm{mb}$ ). However, recent field data taken at very low temperatures $(<190 \mathrm{~K})$ at the tropical tropopause hint at higher $\mathrm{HNO}_{3}$ molar ratios in ice $\left(>10^{-5}\right)$ than predicted by 
our updated trapping model. We have suggested an additional mechanism that may serve to explain these observations. Better understanding of these cold tropical data requires further study.

[68] We have also introduced a possible extension of the updated trapping model that explicitly resolves the physical processes occurring in the ice surface layer instead of merging their net effect into an escape speed. By this means, we have specified the surface-physical conditions in which the updated trapping model is valid. At least for $\mathrm{HNO}_{3}$, our study suggests that such an extension appears to be less important for atmospherically relevant ice particle growth rates. We nevertheless encourage more fundamental laboratory studies aiming at unraveling the true nature of surface processes driving the trace gas uptake for a given ice crystal configuration.

[69] The results presented here can be used to investigate $\mathrm{HNO}_{3}$-ice interactions in cirrus clouds with the help of process or cloud-resolving models that incorporate a full kinetic description of trapping. Situations in which trapping efficiencies and ice particle concentrations are low may necessitate such an approach, as the uptake rate may become kinetically limited. To study the global chemical impact on the partitioning of $\mathrm{NO}_{y}$ species and ultimately on ozone, a simpler parameterization for use in large-scale models might be more appropriate. We will develop such a parameterization scheme in future work.

[70] We recommend applying our updated model for trace gases other than $\mathrm{HNO}_{3}$ only after careful analyses in order to constrain the two open parameters or decide whether a more detailed description is required. This information is necessary to either exclude or confirm the role of trapping for trace gases of interest. We envisage investigating simpler systems relevant to upper tropospheric chemistry, such as nitrous acid $\left(\mathrm{HNO}_{2}\right)$ or hydrogen peroxide $\left(\mathrm{H}_{2} \mathrm{O}_{2}\right)$. Hydrochloric acid $(\mathrm{HCl})$ may also be a target of interest, with potential implications for the chemistry of polar stratospheric clouds and cold tropopause cirrus.

\section{Notation}

$a$ ice particle radius

$\dot{a}$ ice particle growth rate

$e$ ice saturation vapor pressure

$f$ total molecular flux

$k$ escape rate

$k_{L}$ desorption rate

$k_{B} \quad$ Boltzmann constant

$l$ diffusion length scale

$m$ molecular mass

$n$ number density in a given phase

$p$ partial pressure

$s$ ice supersaturation

$t$ time

$u$ mean thermal speed of molecules

$v$ escape speed

$\dot{x}$ ice growth rate in planar flow

$D$ diffusion coefficient

$K$ generalized equilibrium constant

$K_{L} \quad$ Langmuir equilibrium constant

$N$ number of molecules per ice particle

$P$ air pressure
$R$ kinetic rates in the resolved ice surface layer

$T$ air temperature

$\alpha$ deposition coefficient on ice

$\gamma$ uptake coefficient for trapping

$\delta$ depth of (resolved) ice surface layer

$\epsilon$ trapping efficiency

$\theta$ fractional coverage

$\mu$ molar ratio of trace gas in ice

$v$ volume of molecules in ice

$\eta$ number density scaled by ambient value

$\sigma$ area per adsorption site

$\phi$ fraction of molecules in gas or ice phase

Subscripts

$i$ ice phase

$s$ surface layer

$w$ water

+ gas phase close to ice surface layer

- ice phase close to ice surface layer

$\infty$ ambient gas phase

[71] Acknowledgments. This work was funded by the European Commission within the Integrated Project "Stratosphere-Climate Links With Emphasis on the UTLS" (SCOUT-O3). Christiane Voigt was funded, in part, through the Helmholtz Junior Research Group HGF-AEROTROP. The $\mathrm{NO}_{y}$ data from SCOUT and EUPLEX were provided by Hans Schlager. We thank David Fahey and Klaus Gottschaldt for constructive comments.

\section{References}

Abbatt, J. P. D. (1997), Interaction of $\mathrm{HNO}_{3}$ with water-ice surfaces at temperatures of the free troposphere, Geophys. Res. Lett., 24, 14791482.

Abbatt, J. P. D., T. Bartels-Rausch, M. Ullerstam, and T. J. Ye (2008), Uptake of acetone, ethanol, and benzene to snow and ice: Effects of surface area and temperature, Environ. Res. Lett., 3, 045008.

Ammann, M., R. Atkinson, R. A. Cox, J. Crowley, R. Hynes, M. E. Jenkin, M. J. Rossi, J. Troe, and T. Wallington, (2008) Summary of evaluated data for atmospheric heterogeneous processes, IUPAC Subcommittee on Gas Kinetic Data Evaluation for Atmospheric Chemistry, International Union for Pure and Applied Chemistry. (Available at http://www. iupac-kinetic.ch.cam.ac.uk, January)

Cox, R. A., M. A. Fernandez, A. Symington, M. Ullerstam, and J. P. D. Abbatt (2005), A kinetic model for uptake of $\mathrm{HNO}_{3}$ and $\mathrm{HCl}$ on ice in a coated wall flow system, Phys. Chem. Chem. Phys., 7, 3434-3442.

Gierens, K. (1994), The influence of radiation on the diffusional growth of ice crystals, Contr. Atmos. Phys., 67, 181-193.

Hanson, D. R. (1992), The uptake of $\mathrm{HNO}_{3}$ onto ice, NAT, and frozen sulfuric acid, Geophys. Res. Lett., 19, 2063-2066.

Heard, D. E. (Ed.) (2005), Atmospheric chemistry, Faraday Discuss., 130, $241-264$

Huthwelker, T., M. Ammann, and Th. Peter (2006), The uptake of acidic gases on ice, Chem. Rev., 106, 1375-1444.

Kärcher, B., and M. M. Basko (2004), Trapping of trace gases in growing ice crystals, J. Geophys. Res., 109, D22204, doi:10.1029/2004JD005254.

Kärcher, B., and C. Voigt (2006), Formation of nitric acid/water ice particles in cirrus clouds, Geophys. Res. Lett., 33, L08806, doi:10.1029/ 2006 GL025927.

von Kuhlmann, R., and M. G. Lawrence (2006), The impact of ice uptake of nitric acid on atmospheric chemistry, Atmos. Chem. Phys., 6, 225235.

Popp, P. J., et al. (2004), Nitric acid on subtropical cirrus cloud particles, J. Geophys. Res., 109, D06302, doi:10.1029/2003JD004255.

Popp, P. J., et al. (2007), Condensed-phase nitric acid in a tropical subvisible cirrus cloud, Geophys. Res. Lett., 34, L24812, doi:10.1029/ 2007 GL031832.

Pöschl, U., Y. Rudich, and M. Ammann (2007), Kinetic model framework for aerosol and cloud surface chemistry and gas-particle interactions: Part 1. General equations, parameters, and terminology, Atmos. Chem. Phys., 7, 5989-6023.

Pruppacher, H. R., and J. D. Klett (1997), Microphysics of Clouds and Precipitation, Kluwer Acad., Norwell, Mass.

Thibert, E., and F. Dominé (1998), Thermodynamics and kinetics of the solid solution of $\mathrm{HNO}_{3}$ in ice, J. Phys. Chem. B, 102, 4432-4439. 
Ullerstam, M., and J. P. D. Abbatt (2005), Burial of gas phase $\mathrm{HNO}_{3}$ by growing ice surfaces under tropospheric conditions, Phys. Chem. Chem. Phys., 7, 3596-3600.

Ullerstam, M., T. Thornberry, and J. P. D. Abbatt (2005), Uptake of gasphase nitric acid to ice at low partial pressures: Evidence for unsaturated surface coverage, Faraday Discuss., 130, 211-226.

Voigt, C., et al. (2006), Nitric acid in cirrus clouds, Geophys. Res. Lett., 33 , L05803, doi:10.1029/2005GL025159.
Voigt, C., et al. (2007), In situ observations and modeling of small nitric acid-containing ice crystals, Atmos. Chem. Phys., 7, 3373-3383.

J. P. D. Abbatt, Department of Chemistry, University of Toronto, Toronto, ON M5S 3H6, Canada.

R. A. Cox, Department of Chemistry, University of Cambridge, Lensfield Road, Cambridge CB2 1EW, UK.

B. Kärcher and C. Voigt, Deutsches Zentrum für Luft- und Raumfahrt, Institut für Physik der Atmosphäre, Oberpfaffenhofen, D-82234 Wessling, Germany. (bernd.kaercher@dlr.de)

P. J. Popp, Leeds School of Business, UCB 419, Building \#4, Boulder, CO 80309-0419, USA. 